1 University of North Parana UNOPAR, School of Dentistry, Londrina, PR, Brazil

2 Paranaense University - UNIPAR School of Dentistry, Umuarama PR, Brazil
Corresponding author: Murilo Baena Lopes University North of Parana, Rua Marselha, 183, Londrina, PR, 86.040-140, Brazil

E-mail: baenalopes@gmail.com, Tel: +554333717820

Received: June 04, 2018

Accepted: November 19, 2018

\section{Evaluation of physico-chemical and mechanical properties of MTA-based root canal sealer}

Clauber Romagnoli ${ }^{1}$, Klíssia Romero Felizardo², Ricardo Danil Guiraldo ${ }^{1}$, Alcides Gonini Júnior ${ }^{1}$, Renan Hideki Kaneshima', Sandrine Bittencourt Berger ${ }^{1}$, Murilo Baena Lopes ${ }^{1 *}$

Aim: The aim of this study was to evaluate and compare the setting times (ST), flow (FW), radiopacity (RP), dimensional stability (DS), solubility (SB), and polymerization stress (PS) of the MTA Fillapex and AH Plus root canal sealers. Methods: The above qualities were tested according to ISO 6876/2001 standardization. The water used in the dimensional stability test was evaluated to verify the presence of released materials. For the photoelastic analysis, 20 photoelastic resin rings were prepared, and the root canal sealers were inserted. After 24 hours, the specimens were analyzed in a Polariscope. Data of the setting times, flow tests, radiopacity, dimensional stability, and solubility tests were submitted to a Kolgomorov-Smirnov test and then to a Student's t-test at the $5 \%$ significance level. Results: The data derived from photoelastic analyses were submitted to an ANOVA and Tukey's test with a significance level of 5\%. MTA Fillapex and AH Plus complied with ISO 6876/2001. However, there were significant differences $(p<0.05)$ between the two cements for ST, FW, RP, DS, and SB. MTA Fillapex showed higher FW, $\mathrm{SB}$, and PS when compared with AH Plus. Conclusions: MTA Fillapex and AH Plus complied with ISO 6876/2001 in terms of ST, FW, RP, DS, and SB. MTA Fillapex showed higher PS when compared to AH Plus.

Keywords: Dental materials. Endodontics. Surface properties. Root canal obturation. 


\section{Introduction}

In general, the process of filling root canals requires an endodontic sealer and a core material. Currently, the most commonly used core material for filling root canals is the gutta-percha cone, which is universally accepted and regarded as the gold standard in obturation of the root canal system ${ }^{1}$. Nevertheless, the gutta-percha cone cannot adhere to the dentinal surface, so an endodontic sealer should provide good adhesion and flowability that can penetrate into the irregularities and fill the space between the gutta-percha cone and the root canal walls ${ }^{2}$. Therefore, the root canal sealer has as much or more importance than the core material in providing successful clinical outcomes ${ }^{3}$.

Root canal sealers are responsible for different functions during the filling of root canals: they lubricate and guide the settlement of the main cone, serve as a bonding agent between the cones and the canal walls, and fill the anatomic spaces that gutta-percha cannot achieve. In addition, they might have antibacterial properties, which is desirable, especially from a clinical viewpoint, because it is not uncommon that microorganisms flourish in the root canal system after treatment ${ }^{4}$.

The research on adhesive technology has contributed to determining a way to minimize leakage and thereby increase the sealing ability between the filling materials and the root walls ${ }^{5}$. Several sealers have been developed and studied for endodontic treatment. Among them is AH Plus, a resin-based cement that has important properties as a long-term sealant and an appropriate dimensional stability and high radiopacity. Moreover, it has a higher adhesion bond strength when compared with other sealers, and it has good biological properties ${ }^{6,7}$.

In recent years, Mineral Trioxide Aggregate (MTA) has received much recognition among all endodontics materials ${ }^{8}$ not only because of its versatility but also because it can be used to recover teeth that have been deemed as nonrestorable due to extensive caries, developmental anomalies, and iatrogenic damage ${ }^{9}$. It was first described as a material for sealing off all of the pathways of communication between the root canal system and the external surface of the tooth and later as a root-filling material7,10,11. MTA is a powder composed of tricalcium silicate, bismuth oxide, dicalcium silicate, tricalcium aluminum, aluminum-tetracalcium ferric, and dehydrated calcium sulfate, which hardens when hydrated and becomes a colloidal gel with a $\mathrm{pH} 12.5^{12,13}$. In addition, MTA is rich in calcium ions, which are converted into calcium hydroxide in aqueous solutions and improve biocompatibility and promote the action of cementoblasts ${ }^{14}$.

MTA is a bioactive material ${ }^{15}$ that has the ability to stimulate the release of cytokines from bone cells, indicating that it actively promotes hard tissue formation ${ }^{16}$, in addition to its antimicrobial properties and reduced cytotoxic effect ${ }^{17}$. The excellent properties of a mineral trioxide aggregate, including its biocompatibility, bioactivity, and osteoconductivity, have fostered a race to develop new calcium silicate-based endodontic sealers that incorporate MTA ${ }^{18}$. However, both the difficulty of manipulation and the short working time limit its use as a root canal sealer ${ }^{19}$. MTA formulation has been modified by adding polymers and other sub- 
stances to improve properties such as flowability, setting time, and bond strength, without changing its biocompatibility ${ }^{20}$. MTA Fillapex (Angelus, Londrina, PR, Brazil)-which comprises MTA, silica nanoparticles, silicate resin, natural resin, bismuth oxide, and pigments-came to be applied as a new silicate-based sealer ${ }^{21}$. It has suitable flow, good sealing, and low solubility 22,23 and is indicated for use in cold and warm root-filling techniques ${ }^{24}$.

Thus, the purpose of this study was to evaluate and compare the setting times, flow, radiopacity, dimensional stability, solubility, and photoelastic analyses of the MTA Fillapex (Angelus, Londrina, PR, Brazil) and AH Plus (Dentsply-De-Trey, Konstanz, Germany) root canal sealers. The hypothesis was that both materials should exhibit similar behaviors for all properties.

\section{Materials and methods}

\section{Setting Time}

Setting time was evaluated following the International Standards Organization (ISO) specifications $6876: 2001^{25}$. Ten copper circular molds $(n=10)$ with an internal diameter of $10.0 \mathrm{~mm}$ and a thickness of $2.0 \mathrm{~mm}$ were used. The sealers were mixed according to the manufacturer's directions. These molds were maintained at $37 \pm 2{ }^{\circ} \mathrm{C}$ and a relative humidity of $95 \pm 2 \%$. Immediately and at 150 seconds after the onset of mixture, a 1/3-lb Gilmore-type needle with a flat end with a diameter of $2.0 \pm 0.1 \mathrm{~mm}$ was carefully lowered vertically and onto the horizontal surface of each specimen in 15-minute intervals. Next, the 1-lb Gilmore-type needle was used to measure the final setting time. The needle tip was cleaned, and the process was repeated at 60 -second intervals until no indentations were visible. The setting time that each sealer needed to reach this state was noted.

\section{Flow Test}

According to ANSI/ADA specification no. $57^{26}, 0.5 \mathrm{~mL}$ of each cement was placed in the center of a glass plate with a disposable 5-mL syringe. At 180 seconds after the start of mixing, another glass plate was placed centrally on top of the sealer, followed by a weight with a mass of $120 \mathrm{~g}$. Ten minutes after the commencement of mixing, the load was removed, and the major and minor diameters of the compressed discs were measured with a digital caliper (Mitutoyo MTI Corp., Tokyo, Japan). If the major and minor diameter discs were not uniformly circular or did not match within $1 \mathrm{~mm}$, the test was repeated. The test was repeated until 10 cement discs of each group were obtained.

\section{Solubility}

A Teflon ring matrix of $1.0 \mathrm{~mm}$ in height and $15.0 \mathrm{~mm}$ in diameter was made, and 20 impressions were taken with polyvinylsiloxane. These molds were filled with fresh experimental sealers $(n=10)$ and covered with cellophane sheet. Next, the assembly was transferred to an incubator set at $95 \%$ relative humidity and $37^{\circ} \mathrm{C}$, left to stand for a period corresponding to three times the setting time, and was then removed. 
The specimens were removed from the molds and measured, two by two, on a precision balance (JK-180, Chyo Balance Corp., Tokyo, Japan). The specimens were immersed in $7.5 \mathrm{~mL}$ of deionized water for 7 days at $37^{\circ} \mathrm{C}$ in an incubator. Afterward, they were removed, rinsed, and stored in a desiccator for 24 hours and measured. The solubility of the sealer was determined by ascertaining the weight loss of each sample, using the mass difference between the original and the final mass ${ }^{25}$.

\section{Dimensional Stability Test}

A cylindrical Teflon matrix that was $6 \mathrm{~mm}$ in diameter and $12 \mathrm{~mm}$ tall was made, and 20 impressions were taken with polyvinylsiloxane. The molds were fixed with wax in a glass plate and filled with the experimental sealers $(n=10)$ and then covered with a polyester strip. The specimens were measured with a digital caliper (Mitutoyo, South America, São Paulo, Brazil) after they sat in an incubator at $37^{\circ} \mathrm{C}$.

The specimens were immersed in a flask with $2.24 \mathrm{~mL}$ of deionized water and stored in an incubator for 30 days. The specimens were then removed, dried, and measured again to obtain the final specimen length ${ }^{25}$. The water in which the samples were immersed was analyzed in a UV-visible spectrophotometer (BioMate ${ }^{\mathrm{TM}} 3$, Thermo Fisher Scientific, Waltham, Massachusetts, United States) to evaluate the presence of material release.

\section{Radiopacity}

A Teflon mold that was $1 \mathrm{~mm}$ thick and $10 \mathrm{~mm}$ in diameter was made, and 20 impressions were taken with polyvinylsiloxane (Aquasil Ultra Smart Wetting, Dentsply Caulk, Milford, Delaware, United States). The sealers were poured into the impressions matrix on a glass plate covered with a cellophane sheet and another glass plate. The filled impressions were kept at $37^{\circ} \mathrm{C}$ and $100 \%$ of the relative humidity until the cements completely set.

The matrix was removed after the sealer completely set, and the thicknesses of the specimens were confirmed with a digital caliper (Mitutoyo, South America, São Paulo, Brazil). Radiographs were taken with a radiographic unit (Electronic Spectro 70X, Dabi Atlante, Ribeirão Preto, Brazil) with 70 kV, 10 mA, an exposure time of 0.30 seconds, and a focus-film distance of $30 \mathrm{~cm}$ using a specific apparatus composed of two $30-\mathrm{cm}$ plastic rulers and two metal clamps. Along with the specimens, an aluminum step wedge was positioned together to enable the analysis of the radiographic density. The scale comprised 19 aluminum increments with increasing thickness variations of $0.5 \mathrm{~mm}$. Next, the radiographs were processed via a digital radiography system (Schick Technologies Inc., United States).

To determine the radiograph densities, one observer compared the sealer image with the scale. The evaluation was performed three times for each sample, and the average was taken to determine the level of radiopacity of each sample. The higher the level of the scale, the higher the radiopacity of each sealer ${ }^{25}$.

\section{Photoelastic Analysis}

Twenty photoelastic resin rings (PL2, Vishay measurements, Raleigh, United States) were made with the following measurements: a $5-\mathrm{mm}$ internal diameter, a $15-\mathrm{mm}$ 
external diameter, and a 2-mm depth. After the polymerization of the photoelastic resin, the inner walls were sandblasted with $50-\mathrm{mm}$ aluminum oxide particles. The specimens were divided into two groups of 10 each, depending on the tested material. The root canal sealers were manipulated and poured into the photoelastic ring cavities. The specimens were stored at $37^{\circ} \mathrm{C}$ for 24 hours. After this period, the specimens were removed from the incubator and analyzed in a Polariscope (Photostress, Vishay LF/Z-2, Malvern, United States). The data were obtained in MPa via Polariscope software (PSCalc, Vishay Measurements Group Inc., United States).

\section{Statistical Analysis}

Data of the setting time, flow test, radiopacity, dimensional stability, and solubility test were submitted to a nonparametric test, the Kolmogorov-Smirnov test, and then subjected to Student's t-test at the 5\% significance level. The data for the photoelastic analysis were submitted to an ANOVA and Tukey's test with a significance level of $5 \%$.

\section{Results}

\section{Setting Time}

ISO $6876 / 2001^{25}$ specifies that the curing time of a sealer must not be more than $10 \%$ of those stated by the manufacturers. The minimum setting times provided by the manufacturers of AH Plus and MTA Fillapex are 8 hours and 2 hours, respectively.

Table 1 shows the initial and final setting times for each root canal sealer, as well as the comparisons by Student's t-test between the initial and final mean values of setting times. Both root canal sealers were consistent with ISO 6876/2001 standardization.

\section{Flow Test}

Root canal sealers were evaluated in accordance with ANSI/ADA specification no. $57^{26}$, which stipulates that cements should not display diameters less than $20 \mathrm{~mm}$. The only differences between ADA ${ }^{26}$ and ISO 6876/200125 standards are the volume analyzed and the minimum diameters of the spreads. Table 1 contains the mean diameter (in $\mathrm{mm}$ ) of the circles obtained during the flow test. Both tested sealers

Table 1. Physiochemical properties of the investigated sealers.

\begin{tabular}{lcc}
\hline & AH Plus & MTA Fillapex \\
\hline Setting Time (seconds) & & \\
$\quad$ Initial Setting & $677.00 \pm 19.06 \mathrm{a}$ & $255.60 \pm 14.25 \mathrm{~b}$ \\
$\quad$ Final Setting & $694.90 \pm 24.11 \mathrm{a}$ & $504.80 \pm 95.01 \mathrm{~b}$ \\
\hline Flow (mm) & $35.56 \pm 1.03 \mathrm{a}$ & $47.76 \pm 0.65 \mathrm{~b}$ \\
Solubility (\%) & $0.45 \pm 0.14 \mathrm{a}$ & $2.17 \pm 2.24 \mathrm{~b}$ \\
Spectrometric Analysis (\%) & $0.02 \pm 0.00 \mathrm{a}$ & $0.20 \pm 0.02 \mathrm{~b}$ \\
Dimensional Stability (\%) & $0.01 \pm 0.05 \mathrm{a}$ & $-0.42 \pm 1.74 \mathrm{a}$ \\
Radiopacity (mm /\%) & $9.4 \mathrm{~mm} / 18.80 \pm 0.42 \mathrm{a}$ & $6.25 \mathrm{~mm} / 12.50 \pm 1.78 \mathrm{~b}$ \\
Photoelastic analysis (MPa) & $1.05 \pm 1.34 \mathrm{a}$ & $2.05 \pm 1.14 \mathrm{~b}$ \\
\hline
\end{tabular}

Different letters in the same row indicate statistical difference by Student's t test $(p<0.05)$. 
exhibited the minimum diameter requested by ADA/ANSI. However, a statistical analysis revealed a significant difference between AH Plus and MTA Fillapex.

\section{Solubility}

ISO $6876: 2001^{25}$ states that no more than 3 wt \% of a cured root canal sealer should dissolve in water. Each tested sealer's solubility was below this value and was in accordance with ISO 6876/2001 standardization, though MTA Fillapex exhibited a significantly higher solubility than AH Plus. Table 1 showed the percentage of changed weight of the tested specimens.

\section{Dimensional Stability}

ISO $6876: 2001^{25}$ requirements for this test state that the mean linear shrinkage of the sealer shall not exceed $1 \%$ or $0.1 \%$ in expansion. All sealers complied with ISO $6876 / 2001$ standardization. Table 1 shows the MTA Fillapex and the AH Plus specimens' values for dimensional stability in percentages. The Student's t-test showed no statistical difference between MTA Fillapex and AH Plus. The deionized distilled water used for the solubility test of AH Plus and MTA Fillapex was submitted to a UV-visible spectrophotometer, and Table 1 shows the results. The Student's t-test revealed a statistical difference between MTA Fillapex and AH Plus.

\section{Radiopacity}

ISO $6876: 2001^{25}$ stipulates that the radiopacity should be higher than the radiopacity of $3 \mathrm{~mm}$ of aluminum. The tested root canal sealers had values above the ISO regulation. Table 1 shows the means of the radiopacity levels ( $\mathrm{mm}$ of aluminum) for each root canal sealer. The statistical analysis demonstrated that AH Plus significantly had a higher radiopacity than MTA Fillapex.

\section{Photoelastic Analysis}

In the present study, photoelastic analysis was used to determine how the sealers could generate stress on root dentin. Table 1 shows the photoelastic means for the MTA Fillapex and AH Plus specimens. The statistical analysis demonstrated that MTA Fillapex produced a significantly higher strain than AH Plus.

\section{Discussion}

The present study evaluated the physical properties of AHPlus, a two-component-paste root canal cement that is based on the polymerization reaction of epoxy resin amines, and MTA Fillapex, a MTA-based sealer composed of resins, radiopaque bismuth, nanoparticulated silica, and MTA. The physical properties of a material give important information about its applicability. Thus, setting times, flow, solubility, dimensional stability, and radiopacity were measured for each sealer, in terms of ISO 6876/200125. Moreover, a photoelastic analysis was conducted to evaluate how the sealers could generate stress on root dentin. The hypothesis of the study was rejected because the materials showed the same behaviors for all of the measured properties. 
The setting time is the time necessary for the sealer to achieve its definitive properties ${ }^{27}$. It depends on the constituent components, particle size, ambient temperature, and relative humidity ${ }^{28}$. According to ISO $6876: 2001^{25}$, the setting time should not vary by more than $10 \%$ of the manufacturer's information. The minimum setting times provided by the manufacturers of AH Plus and MTA Fillapex are 8 hours and 2 hours, respectively. There is a lack of agreement in the literature about the initial setting time for AH Plus. Different studies show ranges between 8 hours and 20 minutes to 24 hours ${ }^{29,30}$. This variability in setting time might be influenced by the regions where the paste was collect from the tube; for example, higher values for the setting time were found when the sealer was collected from the beginning of the tubes ${ }^{31}$. In the present study, the initial setting of AH Plus was 10 hours and 38 minutes; the final setting was 11 hours and 5 minutes. The mean values for MTA Fillapex was 3 hours and 51 minutes for the initial setting and 7 hours and 56 minutes for the final setting. Therefore, the setting times of both sealers in this study were greater than the minimum values reported by the manufacturers, but they were in accordance with ISO specifications. AH Plus's longer setting time was most likely due to the polymerization process, which is based on the polymerization reaction of epoxy resin amines that occurs gradually ${ }^{32}$. On the other hand, the lower setting time presented by MTA Fillapex can be attributed to the effects of butyl ethylene glycol disalicylate. This chemical compound can be used to obtain resins or polymers with different physical characteristics as rheological characteristics and handling properties. However, further studies are necessary to determine the actual effects of this compound on MTA Fillapex ${ }^{22}$.

A sealer's ability to flow and penetrate into irregularities and accessory canals and between gutta-percha cones is an important property ${ }^{29}$. However, an endodontic sealer must have a moderate flow rate because an excessive flow increases the chance that the material will extrude toward the periapical region, which may compromise periapical healing ${ }^{33}$. In the present study, both of the tested sealers exhibited the minimum diameter requested by ANSI/ADA 57:200026, which is consistent with other studies ${ }^{34-36}$. MTA Fillapex had a significantly higher flow value than that of $\mathrm{AH}$ Plus. In general, factors such as the composition, particle size, shear rate, temperature, and time from mixing are mainly associated with the flowability characteristics of endodontic sealers. Furthermore, the resin to MTA ratio can influence flowability and, perhaps, justify the high flow values of MTA Fillapex ${ }^{22}$.

Solubility is the ability of a substance to dissolve in another, and it is expressed as the concentration of the saturated solution of the former in the latter ${ }^{37}$. According to ISO $6876: 2001^{25}$, no more than 3 wt \% of a cured root canal sealer should dissolve in water. Materials with a higher solubility may release irritants and increase the risk of leakage and bacterial colonization ${ }^{37}$. The solubility of the tested sealers were in agreement with ISO 6876, though MTA Fillapex exhibited a significantly higher solubility than AH Plus. In our study, the results for AH Plus were consistent with those of previous studies ${ }^{32}$; however, in the present study, the range was higher than those of the previous studies. MTA Fillapex presented a lower solubility value when compared with previous studies that reported values between 14.22 and $14.94 \%$. According to Viapiana et al. ${ }^{38}$, MTA Fillapex's high solubility is likely due to the additives that are incorporated into the composition of the sealer, which likely destabi- 
lize its matrix. In addition, the presence of MTA and bismuth trioxide can promote negative effects on solubility. It is important to consider that solubility tests are performed after the sealers are set in a controlled ambience. These characteristics do not occur in clinical conditions in which some degree of humidity inside the root canal may exist, and fresh mixed sealer is used during the obturation procedure. Therefore, solubility in a clinical situation is probably higher ${ }^{37,38}$. Moreover, it is worth mentioning that the disintegration process should be considered as well when the solubility properties are analyzed. Disintegration is the act or effect of falling apart, the separation of something from a whole. When solubility is tested, there is no particle in suspension. However, in disintegration tests, there is a release of particles that remain in suspension ${ }^{37}$. In our study, analyzing the deionized distilled water used for the dimensional stability test resulted in a greater residue release of MTA Fillapex than $\mathrm{AH}$ Plus. This result indicates that the solubility of AH Plus is lower than that of MTA Fillapex. The performance of AH Plus might be related to the characteristics of its resinous matrix, which is more resistant to the solubility ${ }^{3}$.

Dimensional stability demonstrates the shrinkage or expansion of the material following setting. A primary role of a root canal sealer is to act as a physical blockage from invading bacteria originating in the oral cavity. In this perspective, sealers and other components of the filling should ideally be volumetrically stable or increase slightly3,32. The requirements for compliance with the ISO 6876:200125 standard have been set at a linear expansion of not more than $0.1 \%$ or shrinkage of not more than 1\%. In the present study, all sealers complied with the ISO 6876:200125 standardization. AH Plus expanded (0.01\%), and MTA Fillapex contracted linearly $(-0.42 \%)$; these results corroborate previous studies on epoxy resin-based sealers ${ }^{32}$. The expansion of AH Plus can be explained by the water absorption of epoxy resin after polymerization. The polymerization shrinkage of AH Plus occurred when the sample was immersed in distilled water. Possibly, this expansion of AH Plus sealer must compensate for the shrinkage suffered by the resin-based sealer after polymerization ${ }^{32,38}$. On the other hand, the contraction of MTA Fillapex may be related to the high solubility of this sealer ${ }^{38}$.

Root canal sealers should ideally have some degree of radiopacity in order to detect its placement and to be easily distinguishable from dentin and gutta-percha on radiographs. ISO $6876: 2001^{25}$ stipulates that the radiopacity should be higher than the radiopacity of $3 \mathrm{~mm}$ of aluminum. AH Plus and MTA Fillapex were found to be in agreement with ISO 6876:200125. A statistical analysis showed that AH Plus had a significantly higher radiopacity than MTA Fillapex. These results were most likely due to the presence of different radiopacifying agents in each material. AH Plus has zirconium oxide, iron oxide, and calcium tungstate in its composition, which may account for the superior radiopacity ${ }^{38}$. The bismuth trioxide in the MTA Fillapex is responsible for its lower radiopacity ${ }^{38}$.

Only a few studies have used photoelastic stress analyses for evaluating root canal sealers, most likely because this test is not covered by ISO recommendations. However, it is a reliable method for determining how root canal sealers can generate stress in the root dentin. Overall, the intensity of the developed stress is associated with three principal factors: cavity geometry (C-factor), material characteristics, and 
restorative technique ${ }^{39}$. In the present study, AH Plus showed a lower polymerization stress when compared with MTA Fillapex. Apparently, the linear contraction presented by MTA Fillapex generated higher stress than the expansion of AH Plus. In general, if a filling material expands, there is a risk that the root may fracture. The setting expansion of root canal sealers induces radial pressure on the pulpal aspect of the dentin. The risk of fracture is due to the associated tangential strain, the magnitude of which is governed by the elastic modulus of dentin and the filling, the percentage expansion of the filling, and the tensile strength of dentin. Therefore, the consequences of a limited expansion by the sealers are highly dependent on the materials ${ }^{3}$. In addition, the placement of gutta-percha, which is a low-modulus material, reduces the volume of the root canal affected by sealer expansion and can be expected to absorb some of the generated stress ${ }^{3}$. On the other hand, contraction appears to be a less desirable property in these materials. Contraction due to polymerization is not problematic when it occurs on an unbonded surface; however, stress is generated at a cavity interface as a result of the forces generated by the contraction of the composite bonded to the dental structure ${ }^{39}$. Polymerization shrinkage leads to many clinical problems, such as marginal staining, recurrent caries, and restoration failure at the composite or tooth interface ${ }^{39}$. With a requirement of $1 \%$ maximum shrinkage, it only takes a material thickness of $100 \mathrm{~mm}$ to produce a void of $1 \mathrm{~mm}$, which is enough space for bacterial penetration and growth. Thus, an insufficient root filling and shrinking material may be more likely to threaten success than a slightly expanding root canal sealer.

In conclusion, MTA Fillapex and AH Plus complied with ISO 6876:200125, in terms of setting times, flow, solubility, dimensional stability, and radiopacity. MTA Fillapex showed a higher flow profile, solubility, and polymerization stress when compared with AH Plus. Further studies should aim to obtain more insight into the physical, mechanical, and chemical properties of the root canal sealer and how best to use them in specific clinical procedures.

\section{References}

1. Kaya BU, Kececi AD, Belli S. Evaluation of the sealing ability of gutta-percha and thermoplastic synthetic polymer-based systems along the root canals through the glucose penetration model. Oral Surg Oral Med Oral Pathol Oral Radiol Endod. 2007 Dec;104(6):e66-73. doi: 10.1016/j.tripleo.2007.06.024.

2. Skinner RL, Himel VT. The sealing ability of injection-molded thermoplasticized gutta-percha with and without the use of sealers. J Endod. 1987 Jul;13(7):315-7. doi: 10.1016/S0099-2399(87)80112-7.

3. Orstavik D, Nordahl I, Tibballs JE. Dimensional change following setting of root canal sealer materials. Dent Mater. 2001 Nov;17(6):512-9.

4. Gjorgievska E, Apostolska S, Dimkov A, Nicholson JW, Kaftandzieva A. Incorporation of antimicrobial agents can be used to enhance the antibacterial effect of endodontic sealers. Dent Mater. 2013 Mar;29(3):e29-34. doi: 10.1016/j.dental.2012.10.002.

5. Tay FR, Loushine RJ, Weller RN, Kimbrough WF, Pashley DH, Mak YF, et al. Ultrastructural evaluation of the apical seal in roots filled with a polycaprolactone-based root canal filling material. J Endod. 2005 Jul;31(7):514-9. 
6. Nagas E, Cehreli Z, Uyanik MO, Durmaz V. Bond strength of a calcium silicate-based sealer tested in bulk or with different main core materials. Braz Oral Res. 2014 28(1):1-7. doi: 10.1590/1807-3107BOR-2014.vol28.0046.

7. Torabinejad M, Watson TF, Pitt Ford TR. Sealing ability of a mineral trioxide aggregate when used as a root end filling material. J Endod. 1993 Dec;19(12):591-5. doi: 10.1016/S0099-2399(06)80271-2.

8. Post LK, Lima FG, Xavier CB, Demarco FF, Gerhardt-Oliveira M. Sealing ability of MTA and amalgam in different root-end preparations and resection bevel angles: an in vitro evaluation using marginal dye leakage. Braz Dent J. 2010 21(5):416-9.

9. Abdo SB, Masudi SM, Luddin N, Husien A, Khamis MF. Fracture resistance of over-flared root canals filled with MTA and resin-based material: an in vitro study. Br J Oral Sci. 2012 11(4):451-7.

10. Bidar M, Sadeghalhoseini N, Forghani M, Attaran N. Effect of the smear layer on apical seals produced by two calcium silicate-based endodontic sealers. J Oral Sci. 2014 Sep;56(3):215-9.

11. Utneja S, Nawal RR, Talwar S, Verma M. Current perspectives of bio-ceramic technology in endodontics: calcium enriched mixture cement - review of its composition, properties and applications. Restor Dent Endod. 2015 Feb;40(1):1-13. doi: 10.5395/rde.2015.40.1.1

12. Schwartz RS, Mauger M, Clement DJ, Walker WA, 3rd. Mineral trioxide aggregate: a new material for endodontics. J Am Dent Assoc. 1999 Jul;130(7):967-75.

13. Kim J, Song YS, Min KS, Kim SH, Koh JT, Lee BN, et al. Evaluation of reparative dentin formation of ProRoot MTA, Biodentine and BioAggregate using micro-CT and immunohistochemistry. Restor Dent Endod. 2016 Feb;41(1):29-36. doi: 10.5395/rde.2016.41.1.29

14. Goncalves JL, Viapiana R, Miranda CE, Borges AH, Cruz Filho AM. Evaluation of physico-chemical properties of Portland cements and MTA. Braz Oral Res. 2010 Jul-Sep;24(3):277-83.

15. Dianat O, Mashhadiabbas F, Ahangari Z, Saedi S, Motamedian SR. Histologic comparison of direct pulp capping of rat molars with MTA and different concentrations of simvastatin gel. J Oral Sci. 2018 Feb 26;doi: 10.2334/josnusd. 16-0690.

16. Torabinejad M, Hong CU, Lee SJ, Monsef M, Pitt Ford TR. Investigation of mineral trioxide aggregate for root-end filling in dogs. J Endod. 1995 Dec;21(12):603-8. doi: 10.1016/S0099-2399(06)81112-X.

17. Osorio RM, Hefti A, Vertucci FJ, Shawley AL. Cytotoxicity of endodontic materials. J Endod. 1998 Feb;24(2):91-6. doi: 10.1016/S0099-2399(98)80084-8

18. Collado-Gonzalez M, Tomas-Catala CJ, Onate-Sanchez RE, Moraleda JM, Rodriguez-Lozano FJ. Cytotoxicity of GuttaFlow Bioseal, GuttaFlow2, MTA Fillapex, and AH Plus on Human Periodontal Ligament Stem Cells. J Endod. 2017 May;43(5):816-822. doi: 10.1016/j.joen.2017.01.001.

19. Camilleri J. Modification of mineral trioxide aggregate. Physical and mechanical properties. Int Endod J. 2008 Oct;41(10):843-9. doi: 10.1111/j.1365-2591.2008.01435.x.

20. Camilleri J. Evaluation of selected properties of mineral trioxide aggregate sealer cement. J Endod. 2009 Oct;35(10):1412-7. doi: 10.1016/j.joen.2009.07.008.

21. Jafari F, Jafari S. Composition and physicochemical properties of calcium silicate based sealers: A review article. J Clin Exp Dent. 2017 Oct;9(10):e1249-e1255. doi: 10.4317/jced.54103.

22. Vitti RP, Prati C, Sinhoreti MA, Zanchi CH, Souza ESMG, Ogliari FA, et al. Chemical-physical properties of experimental root canal sealers based on butyl ethylene glycol disalicylate and MTA. Dent Mater. 2013 Dec;29(12):1287-94. doi: 10.1016/j.dental.2013.10.002.

23. Gandolfi MG, Siboni F, Prati C. Properties of a novel polysiloxane-guttapercha calcium silicate-bioglass-containing root canal sealer. Dent Mater. 2016 May;32(5):e113-26. doi: $10.1016 /$ j.dental.2016.03.001. 
24. Viapiana R, Guerreiro-Tanomaru JM, Tanomaru-Filho M, Camilleri J. Investigation of the effect of sealer use on the heat generated at the external root surface during root canal obturation using warm vertical compaction technique with System B heat source. J Endod. 2014 Apr;40(4):555-61. doi: 10.1016/j.joen.2013.09.026.

25. Standardization IOf. ISO 6876 - Dental Root Canal Sealing Materials. Geneva: International Organization for Standardization; 2001.

26. ANSIADAS. Specification \#57 - Endodontic Sealing Materials. 2000.

27. Batchelor RF, Wilson AD. Zinc oxide-eugenol cements. I. The effect of atmospheric conditions on rheological properties. J Dent Res. 1969 Sep-Oct;48(5):883-7.

28. Uhrich JM, Moser JB, Heuer MA. The rheology of selected root canal sealer cements. J Endod. 1978 Dec;4(12):373-9. doi: 10.1016/S0099-2399(78)80212-X.

29. McMichen FR, Pearson G, Rahbaran S, Gulabivala K. A comparative study of selected physical properties of five root-canal sealers. Int Endod J. 2003 Sep;36(9):629-35.

30. Nielsen BA, Beeler WJ, Vy C, Baumgartner JC. Setting times of Resilon and other sealers in aerobic and anaerobic environments. J Endod. 2006 Feb;32(2):130-2. doi: 10.1016/j.joen.2005.10.024.

31. Baldi JV, Bernardes RA, Duarte MA, Ordinola-Zapata R, Cavenago BC, Moraes JC, et al. Variability of physicochemical properties of an epoxy resin sealer taken from different parts of the same tube. Int Endod J. 2012 Oct;45(10):915-20. doi: 10.1111/j.1365-2591.2012.02049.x.

32. Versiani MA, Carvalho-Junior JR, Padilha MI, Lacey S, Pascon EA, Sousa-Neto MD. A comparative study of physicochemical properties of AH Plus and Epiphany root canal sealants. Int Endod J. 2006 Jun;39(6):464-71. doi: 10.1111/j.1365-2591.2006.01105.x.

33. Sjogren U, Hagglund B, Sundqvist G, Wing K. Factors affecting the long-term results of endodontic treatment. J Endod. 1990 Oct;16(10):498-504. doi: 10.1016/S0099-2399(07)80180-4.

34. Bernardes RA, de Amorim Campelo A, Junior DS, Pereira LO, Duarte MA, Moraes IG, et al. Evaluation of the flow rate of 3 endodontic sealers: Sealer 26, AH Plus, and MTA Obtura. Oral Surg Oral Med Oral Pathol Oral Radiol Endod. 2010 Jan;109(1):e47-9. doi: 10.1016/j.tripleo.2009.08.038.

35. Tanomaru-Filho M, Bosso R, Viapiana R, Guerreiro-Tanomaru JM. Radiopacity and flow of different endodontic sealers. Acta Odontol Latinoam. 2013 26(2):121-5.

36. Camargo RV, Silva-Sousa YTC, Rosa R, Mazzi-Chaves JF, Lopes FC, Steier L, et al. Evaluation of the physicochemical properties of silicone- and epoxy resin-based root canal sealers. Braz Oral Res. 2017 Aug 21;31(e72. doi: 10.1590/1807-3107BOR-2017.vol31.0072.

37. Sousa-Neto MD, Guimaraes LF, Saquy PC, Pecora JD. Effect of different grades of gum rosins and hydrogenated resins on the solubility, disintegration, and dimensional alterations of Grossman cement. J Endod. 1999 Jul;25(7):477-80. doi: 10.1016/S0099-2399(99)80284-2.

38. Viapiana R, Flumignan DL, Guerreiro-Tanomaru JM, Camilleri J, Tanomaru-Filho M. Physicochemical and mechanical properties of zirconium oxide and niobium oxide modified Portland cement-based experimental endodontic sealers. Int Endod J. 2014 May;47(5):437-48. doi: 10.1111/iej.12167.

39. Lopes MB, Valarini N, Moura SK, Guiraldo RD, Gonini Junior A. Photoelastic analysis of stress generated by a silorane-based restoration system. Braz Oral Res. 2011 Jul-Aug;25(4):302-6. 